\title{
Internal mammary lymph node siliconoma in absence of prosthesis rupture: a case series that raises concern for potential risk of overdiagnosis
}

\author{
Valentina Errico $^{1}$, Gianluca Perroni ${ }^{1,2}$, Flavio Milana ${ }^{2,3} \wedge$, Andrea Vittorio Emanuele Lisa ${ }^{4}$, \\ Emilia Marrazzo $^{5}$, Marco Klinger ${ }^{4,6}$, Corrado Tinterri ${ }^{2,5}$, Alberto Testori ${ }^{1}$ \\ ${ }^{1}$ Division of Thoracic Surgery, IRCCS Humanitas Research Hospital, Rozzano, Milan, Italy; ${ }^{2}$ Department of Biomedical Sciences, Humanitas \\ University, Pieve Emanuele, Milan, Italy; ${ }^{3}$ IRCCS Humanitas Research Hospital, Rozzano, Milan, Italy; ${ }^{4}$ Plastic Surgery Department, IRCCS \\ Humanitas Research Hospital, Rozzano, Milan, Italy; ${ }^{5}$ Breast Unit Department, IRCCS Humanitas Research Hospital, Rozzano, Milan, Italy; \\ ${ }^{6}$ Department of Medical Biotechnology and Translational Medicine Biometra, Reconstructive and Aesthetic Plastic Surgery School, University of \\ Milan, Milan, Italy \\ Contributions: (I) Conception and design: V Errico, A Lisa; (II) Administrative support: M Klinger, C Tinterri, A Testori; (III) Provision of study \\ materials or patients: V Errico, A Lisa, E Marrazzo; (IV) Collection and assembly of data: F Milana, G Perroni; (V) Data analysis and interpretation: \\ F Milana, G Perroni; (VI) Manuscript writing: All authors; (VII) Final approval of manuscript: All authors. \\ Correspondence to: Gianluca Perroni, MD. Humanitas Clinical and Research Hospital, Thoracic Surgery Department, 20089, Via Alessandro Manzoni \\ 56, Rozzano, Milan, Italy. Email: gianlucaperroni91@gmail.com.
}

Background Silicon migration after rupture is an adverse event of breast implant, whose risk increases with the aging of prosthesis. The exact prevalence of this complication remains unclear and reported data are inconsistent. In addition, microscopic diffusion of silicone gel through intact implant, known as gel bleeding, might verify thus complicating diagnosis. Although high cohesive gel has reduced the occurrence of gel bleeding, this phenomenon is still possible and its occurrence rate remains underestimated. If silicon droplets migrate in locoregional lymph node, a swelling that mimics recurrence can arise. Therefore, a risk of overdiagnosis is possible when clinicians rely only on imaging techniques. The aim of this study is to evaluate the actual prevalence of metastasis in internal mammary lymph node (IMLN) in presence of PET positive uptake and no prosthesis rupture.

Methods: We retrospectively evaluated our patient's records and selected those with intact breast implants and suspected relapse in IMLN that underwent biopsy, either surgical or imaging guided. All patients performed PET/CT scan showing pathological uptake in IMLNs. A breast magnetic resonance (MRI) or ultrasound (US) imaging confirmed a suspicious adenopathy and excluded prosthesis rupture. From 2015 to 2019 a total of nine patients underwent biopsy of the IMLN and only six of them met inclusion criteria.

Results: Four biopsies were CT-guided, two were surgical. Three patients (50\%) were diagnosed with breast cancer relapse while two (33.3\%) were found with siliconoma and one (16.7\%) was inflammatory.

Conclusions: Siliconoma can occur even without evidence of capsule rupture, challenging the clinicians and leading to a risk of relapse over diagnosis. Echographic, MRI and nuclear medicine imaging criteria may be not sufficient in differential diagnosis. To overcome the issue, we suggest introducing into the clinical practice the biopsy of suspicious enlarged IMLN with minimally invasive technique.

Keywords: Siliconoma; breast cancer; breast implant; internal mammary lymph node (IMLN); case series

Submitted Nov 29, 2020. Accepted for publication May 11, 2021.

doi: 10.21037 /gs-20-860

View this article at: https://dx.doi.org/10.21037/gs-20-860

\footnotetext{
^ ORCID: 0000-0003-3570-9573.
} 


\section{Introduction}

\section{Background}

Siliconoma is a foreign body reaction caused by migration of silicone particles to surrounding or distant tissues, potentially affecting also the lymph nodes. Breast implant rupture represents the main cause of this phenomenon, with a $15 \%$ of occurrence between the third and tenth year after surgery (1). Together with the rupture, microscopic silicone droplets can filter through intact implant surface, a phenomenon known as "gel bleeding". Use of high cohesive gel apparently solved this issue reaching better aesthetical result together with the maintenance of shape and upper pole fill in comparison to older gel prosthesis. Indeed, no gel bleeding has been reported since the introduction $(2,3)$. Nevertheless, gel bleed has been demonstrated to occur also in high cohesive implants in experimental model. Lourenço et al. showed that a passage of silicone is present $\left(1.0444 \mathrm{mg} / \mathrm{cm}^{2} /\right.$ week in smooth models and $0.3377 \mathrm{mg} / \mathrm{cm}^{2} /$ week in textured one) after conventional sterilization processes (4). In 2006, Prantl demonstrated the presence of silicone particles inside vacuolated macrophages contained in capsular and pericapsular tissue of 24 female patients with high cohesive implants (5). Despite magnetic resonance has the highest sensitivity and specificity in diagnosing the prosthesis rupture, the vast majority of gel bleed cases are undetectable even with this technique $(6,7)$.

Routine biopsy of internal mammary lymph node (IMLN) during extended radical mastectomy was abandoned in 1970s mainly because of high complication rate and increased operative time with no survival benefit $(8,9)$. Moreover, isolated IMLN involvement occurs only in $0.1 \%$ of cases and therefore is extremely rare (10). For those reasons, assessment of IMLN had a decreasing role in breast cancer staging evaluation. According to the latest clinical practice guidelines from the National Comprehensive Cancer Network (NCCN) if biopsy cannot be safely obtained but clinical evidence is strongly supportive of recurrence, treatment may commence based on receptor status of the primary tumor $(11,12)$. Thus, it is clear how a diagnostic pitfall may arise if newly isolate IMLN node swelling is detected during breast cancer follow-up and no histological diagnosis is performed. Here, we reviewed our internal patient records and selected those women diagnosed with breast cancer who underwent mastectomy and subsequent prosthesis implant. We highlight that patients who relapsed with a siliconoma diagnosis were scanned with MRI to confirm implant integrity. We arise a diagnostic dilemma in the evaluation of breast cancer relapse since a consistent proportion of patients in our hospital were diagnosed with a siliconoma, which should be a rare adverse event when breast implant is intact.

\section{Objectives}

We aim to estimate the actual prevalence of metastatic IMLN adenopathy in presence of intact high cohesive breast implant and suspicious node swelling at imaging in patients with previous breast cancer experienced in our hospital. The main objective of this study is to explore the concrete risk of incurring in nodal relapse overdiagnosis revising the cases in which histological specimen was obtained. We present the following article in accordance with the AME Case Series reporting checklist (available at https://dx.doi.org/10.21037/gs-20-860) (13).

\section{Methods}

In this retrospective observational study, we analyzed our internal electronic patient records and selected those with radiological suspected relapse in IMLN who previously underwent mastectomy and subsequent prosthesis implant for breast cancer. Exclusion criteria were prosthesis substitution or rupture, metastasis from other tumor and incomplete data with a threshold of $90 \%$. Absence of prosthesis rupture was verified using magnetic resonance (MRI) or ultrasound (US) imaging techniques. From 2015 to 2019 we collected nine patients, three were excluded while six met our criteria. All of the selected patients underwent mastectomy with subsequent reconstruction using high cohesive gel breast implants between 1998 and 2017, three were bilateral.

During follow up, a PET positive IMLN swelling without evidence of prosthesis rupture (Figure 1) was noted.

The study was conducted in accordance with the Declaration of Helsinki (as revised in 2013). This retrospective study was approved by Institutional Review Board of Humanitas clinical and research hospital (approval No. BSIL1) and informed consent was obtained from each patient enrolled.

\section{Statistical analysis}

Due to few number of patients, only descriptive analysis was performed. All statistical analyses were conducted using 


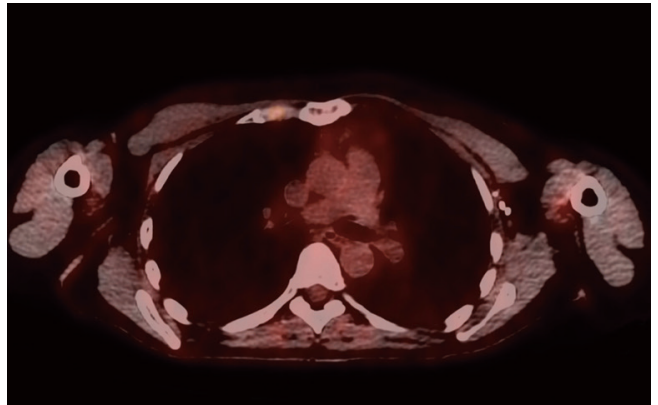

Figure 1 PET/CT scan showing pathological uptake of IMLN. PET/CT, positron emission tomography/computed tomography; IMLN, internal mammary lymph node.

IBM SPSS Statistics V22.0.

\section{Results}

Clinical characteristics of patients are reported in Table 1, laboratory results in Table 2. Mean age at diagnosis of IMLN adenopathy was $47 \pm 7.15$ months and mean time from surgery to biopsy was $84.21 \pm 82.83$ months. All of the patients were asymptomatic. Four biopsies were CTguided (Figure 2), while two were performed under general anesthesia. Only three out of six specimens were metastatic (50\%), two were siliconomas (33.3\%) (Figure 3) and the remaining presented a reactive lymphoid hyperplasia that was consistent with nonspecific inflammatory reaction $(16.7 \%)$. Of note, all the patients with histological diagnosis of siliconoma previously underwent MRI scan that confirmed implant integrity (Figure 4).

\section{Discussion}

CT/PET scan and MRI are routinely used to assess tumor recurrence with sensitivity, specificity and accuracy values of $100 \%, 72 \%, 84 \%$ and $79 \%, 94 \%, 88 \%$, respectively (14). However, low specificity of CT/PET scan may lead to false positive due to other non-neoplastic causes such as nonspecific inflammation or silicone migration in lymph nodes. Based on the available literature, differential diagnosis between relapse and foreign body reaction is easily possible with MRI in presence of either implant rupture or visible extravasation of silicone material nearby prosthesis plus lymph node swelling $(15,16)$. Nevertheless microscopic silicone leakage through intact outer shell occurs, which is usually referred as "gel bleeding". It represents an underestimated complication of breast prosthesis, mainly because it results undetectable at MRI if is not extensive in surrounding tissue $(6,7,17)$. Indeed Di Benedetto et al. in 2006 compared three different imaging technique for detection of implant rupture, including MRI. Surgical removal was performed in eighty-two implants and those with silicone gel layer outside prosthesis without envelope rupture were categorized as "normal", suggesting that gel bleeding is not reported as frequent as it should be (18).

Moreover, there are some evidences of silicone migration to regional lymph node even when macroscopic rupture is not evident. In 2005, Katzin et al. obtained biopsies of regional lymph nodes from 96 patients, including IMLN, and compared histological results with samples from 12 patients without breast implant. As result, 91\% of patients with breast implant had evidence of silicone adenopathy in comparison of none in control group. Interestingly, only $49 \%$ of implants had visible evidence of rupture (19). However, time from insertion of first known implant ranged from 1 to 30 years and therefore non-high cohesive gel models may be included. In addition, lymph nodes were analyzed as a whole and no information on single stations are available. With the introduction of new cohesive gel implants, gel bleeding has not been found (2). Nevertheless, in 2006 Prantl et al. demonstrated the presence of vacuolated macrophages containing silicone in capsules even with new generations prosthesis (5).

Diagnosis of silicone-induced adenopathy through MRI is held with sensitivity and specificity of $20 \%$ and $100 \%$, respectively. In this complex scenario, false negative frequently are identified (20), therefore damaging patient's management. Our results confirm these findings, as both patients with silicone-induced adenopathy performed MRI that did not ruled out the complication.

According to the latest European Society for Medical Oncology (ESMO) guidelines, biopsy of relapsing lesion should be carried out when technically feasible (21). Therefore is possible to assume that in case of impossibility to perform biopsy, other diagnostic methods for breast cancer relapse should be applied. Surgical biopsy of IMLN can be performed through the previous mastectomy incision or, when not possible, using an additional one over related intercostal space, as described by Sacchini et al. (22). Having said that, presence of prosthesis may lead to extremely complex procedure with high risk of rupture or damage to breast implant. Moreover, when additional incision is needed, the aesthetical result is compromised. However image-guided biopsy overcome these limitations, it comes 
Table 1 Clinical characteristics of patients

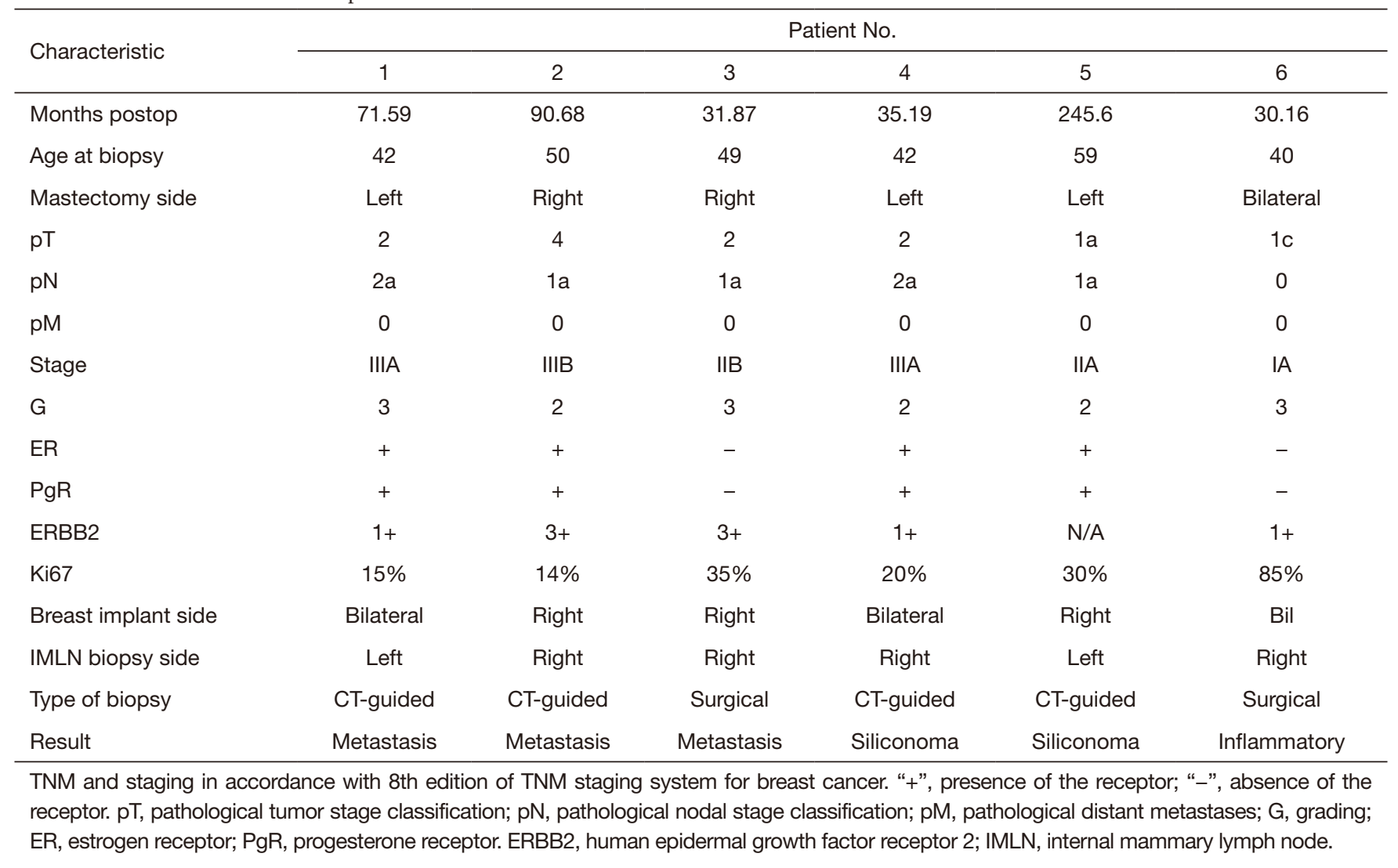

Table 2 Laboratory results of patients

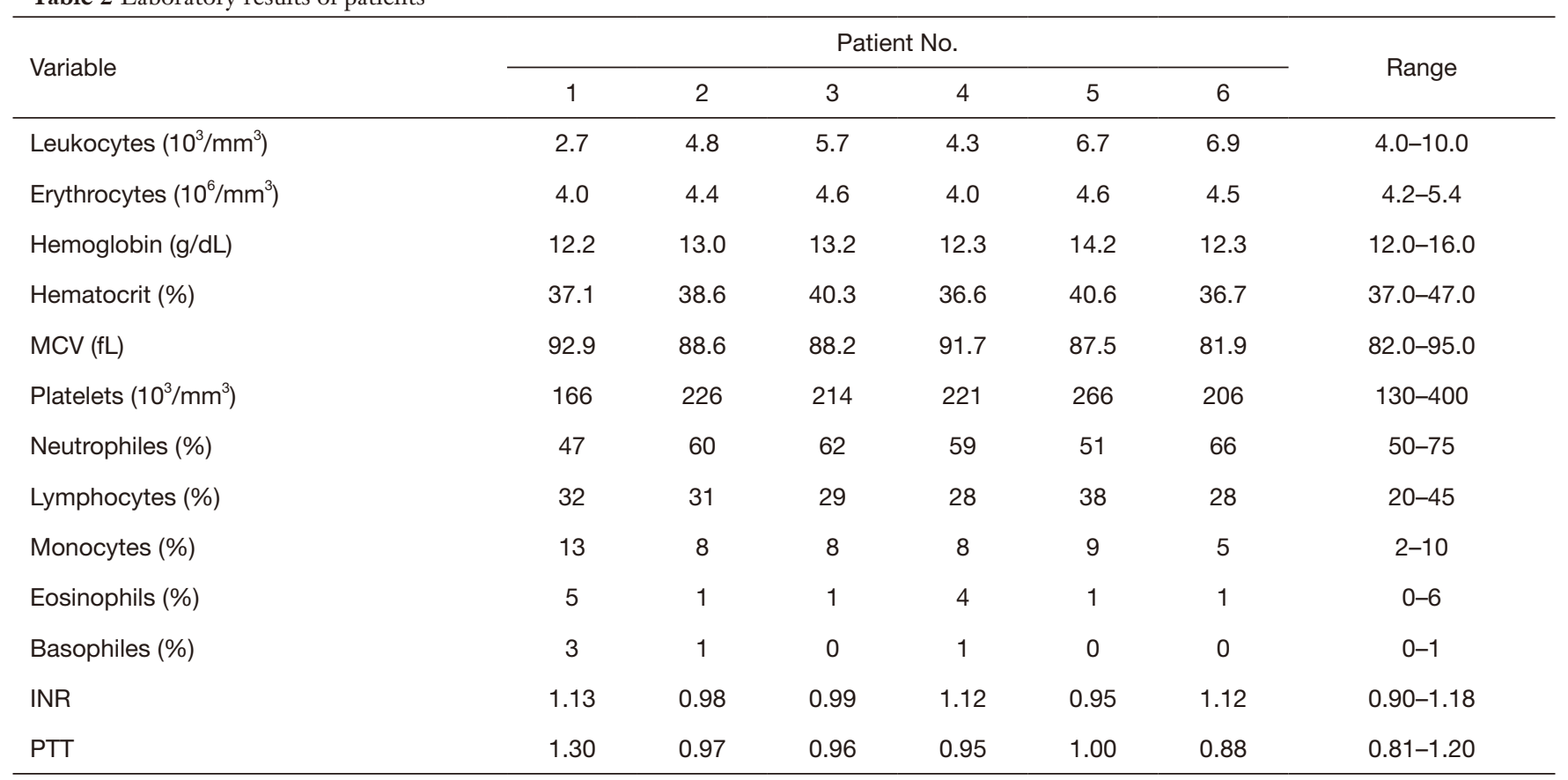

MCV, mean corpuscular volume; INR, international normalized ratio; PTT, partial thromboplastin time. 


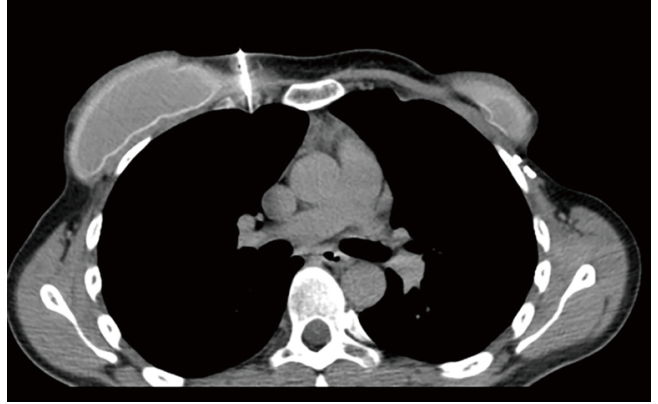

Figure 2 CT-guided biopsy. All of our patients experienced no complications after procedure. CT, computed tomography.

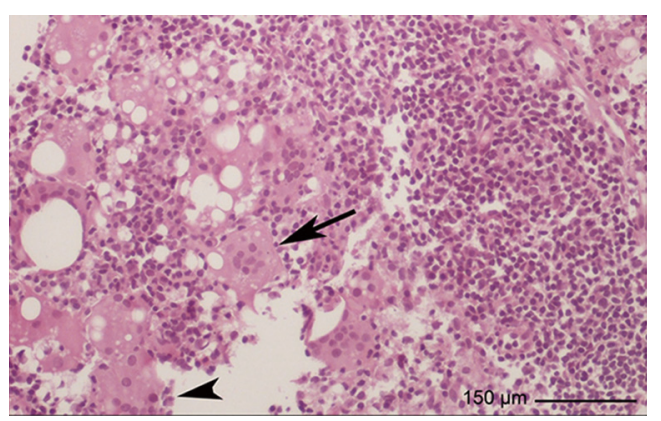

Figure 3 Histologic examination at hematoxylin and eosin staining revealed the presence of Langhans giant cells (black arrow) and foreign-body giant cells (arrowhead) typical of a foreign body granuloma.

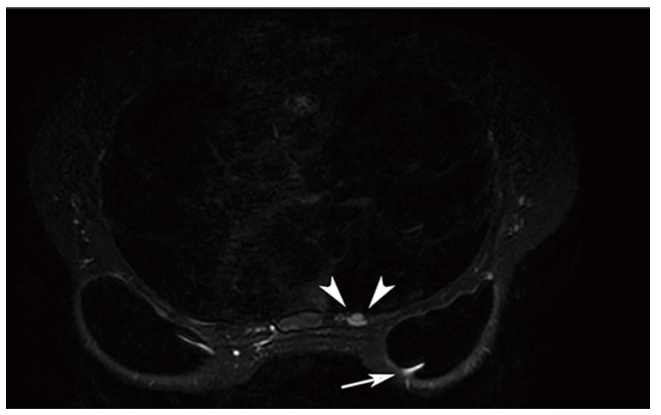

Figure 4 MRI with enlarged internal mammary lymph node (double arrowhead) and normal breast implant with radial folding (white arrow). MRI, magnetic resonance imaging.

with high risk of pleural $(5.1 \%)$ or internal mammary vessels lesion $(9.1 \%)(23)$. In addition, this anatomical site is affected as isolated secondary site in $0.1 \%$ of cases, with no more than $5 \%$ of all breast cancers reported having it as exclusive draining site (10).
These data confirm the trend of clinicians to extensively make use of imaging techniques to diagnose breast cancer recurrence occurring in infrequent and difficult to access site as IMLN.

Based on this information, is clear how a CT/PET positive lymph node swelling may be misinterpreted as relapse in a patient who underwent mastectomy with subsequent breast implant and no evidence of rupture at MRI unless further histological examination is performed. According to our findings, only 50\% of IMLN swelling was metastatic and this result is consistent with a previous finding from Long et al. in 2011. They performed biopsy using video-assisted thoracoscopic surgery (VATS) and found that $40 \%$ of biopsies were positive (24). Interestingly this author suggested a minimally invasive technique with extremely low complication rate leading to stage migration, thus allowing proper treatment. In order to reduce trauma from general anesthesia and double lumen-ventilation, a tubeless technique with loco-regional anesthesia may be feasible (25).

To our knowledge, this is the first evidence in literature describing silicone-based IMLN adenopathy without evidence of rupture in new cohesive gel implants. Although high sensitivity, CT/PET scan may lead to false-positive uptake in siliconoma of lymph nodes due to low specificity of this technique. MRI is the gold standard for the diagnosis of prosthesis rupture, however capability in detecting gel bleed is limited and therefore underestimation of this complication occurs. Even if designed to limit the phenomenon, passage of microscopical silicone droplet trough intact shell in cohesive gel implants is possible and subsequent spread to lymph node may occurs. Current guidelines allow physicians to diagnose and treat recurrence in absence of pathologically proven histology, but even a high clinical suspicion is not a certainty and biopsy should be performed to avoid unnecessary therapies. Nowadays diagnosis can be safely performed using minimally invasive procedure such as VATS or imaging-guided biopsy. The evidence of this study, however, is limited by the retrospective nature and the small sample used. Therefore, further research is needed to clarify this phenomenon.

\section{Acknowledgments}

Funding: None.

\section{Footnote}

Reporting Checklist: The authors have completed the AME 
Case Series reporting checklist. Available at https://dx.doi. org/10.21037/gs-20-860

Data Sharing Statement: Available at https://dx.doi. org/10.21037/gs-20-860

Conflicts of Interest: All authors have completed the ICMJE uniform disclosure form (available at https://dx.doi. org/10.21037/gs-20-860). The authors have no conflicts of interest to declare.

Ethical Statement: The authors are accountable for all aspects of the work in ensuring that questions related to the accuracy or integrity of any part of the work are appropriately investigated and resolved. The study was conducted in accordance with the Declaration of Helsinki (as revised in 2013). This retrospective study was approved by Institutional Review Board of Humanitas clinical and research hospital (approval No. BSIL1) and informed consent was obtained from each patient enrolled.

Open Access Statement: This is an Open Access article distributed in accordance with the Creative Commons Attribution-NonCommercial-NoDerivs 4.0 International License (CC BY-NC-ND 4.0), which permits the noncommercial replication and distribution of the article with the strict proviso that no changes or edits are made and the original work is properly cited (including links to both the formal publication through the relevant DOI and the license). See: https://creativecommons.org/licenses/by-nc-nd/4.0/.

\section{References}

1. Hölmich LR, Friis S, Fryzek JP, et al. Incidence of silicone breast implant rupture. Arch Surg 2003;138:801-6.

2. Colombo G, Ruvolo V, Stifanese R, et al. Prosthetic breast implant rupture: imaging--pictorial essay. Aesthetic Plast Surg 2011;35:891-900.

3. Brown MH, Shenker R, Silver SA, et al. Cohesive silicone gel breast implants in aesthetic and reconstructive breast surgery. Plast Reconstr Surg 2005;116:768-79; discussion 780-1.

4. Loureno FR, Kikuchi IS, Andreoli Pinto T de J. Silicone Gel Bleed on Breast Implants. Open Biomater J 2011;3:14-7.

5. Prantl L, Schreml S, Fichtner-Feigl S, et al. Histological and immunohistochemical investigations with capsular contracture after breast augmentation with smooth silicone gel implants. Handchir Mikrochir Plast Chir 2006;38:224-32.

6. Juanpere S, Perez E, Huc O, et al. Imaging of breast implants-a pictorial review. Insights Imaging 2011;2:653-70.

7. Lindenblatt N, El-Rabadi K, Helbich TH, et al. Correlation between MRI results and intraoperative findings in patients with silicone breast implants. Int $\mathbf{J}$ Womens Health 2014;6:703-9.

8. Veronesi U, Marubini E, Mariani L, et al. The dissection of internal mammary nodes does not improve the survival of breast cancer patients. 30-year results of a randomised trial. Eur J Cancer 1999;35:1320-5.

9. Lacour J, Bucalossi P, Cacers E, et al. Radical mastectomy versus radical mastectomy plus internal mammary dissection. Five-year results of an international cooperative study. Cancer 1976;37:206-14.

10. Fowble B, Solin LJ, Schultz DJ, et al. Frequency, sites of relapse, and outcome of regional node failures following conservative surgery and radiation for early breast cancer. Int J Radiat Oncol Biol Phys 1989;17:703-10.

11. Gradishar WJ, Anderson BO, Abraham J, et al. Breast Cancer, Version 3.2020, NCCN Clinical Practice Guidelines in Oncology. J Natl Compr Canc Netw 2020;18:452-78.

12. Palumbo R, Sottotetti F, Riccardi A, et al. Which patients with metastatic breast cancer benefit from subsequent lines of treatment? An update for clinicians. Ther Adv Med Oncol 2013;5:334-50.

13. von Elm E, Altman DG, Egger M, et al. The Strengthening the Reporting of Observational Studies in Epidemiology (STROBE) statement: guidelines for reporting observational studies. J Clin Epidemiol 2008;61:344-9.

14. Goerres GW, Michel SC, Fehr MK, et al. Follow-up of women with breast cancer: comparison between MRI and FDG PET. Eur Radiol 2003;13:1635-44.

15. Bauer PR, Krajicek BJ, Daniels CE, et al. Silicone breast implant-induced lymphadenopathy: 18 Cases. Respir Med CME 2011;4:126-30.

16. Hurwitz R. F-18 FDG positron emission tomographic imaging in a case of ruptured breast implant: inflammation or recurrent tumor? Clin Nucl Med 2003;28:755-6.

17. Yang N, Muradali D, et al. The augmented breast: a pictorial review of the abnormal and unusual. AJR Am J Roentgenol 2011;196:W451-60.

18. Di Benedetto G, Cecchini S, Grassetti L, et al. Comparative study of breast implant rupture using mammography, sonography, and magnetic resonance 
imaging: correlation with surgical findings. Breast J 2008;14:532-7.

19. Katzin WE, Centeno JA, Feng LJ, et al. Pathology of lymph nodes from patients with breast implants: a histologic and spectroscopic evaluation. Am J Surg Pathol 2005;29:506-11.

20. Klang E, Yosepovich A, Krosser A, et al. Detection of Pathologically Proven Silicone Lymphadenopathy: Ultrasonography Versus Magnetic Resonance Imaging. J Ultrasound Med 2018;37:969-75.

21. Cardoso F, Harbeck N, Fallowfield L, et al. Locally recurrent or metastatic breast cancer: ESMO Clinical Practice Guidelines for diagnosis, treatment and followup. Ann Oncol 2012;23 Suppl 7:vii11-9.

Cite this article as: Errico V, Perroni G, Milana F, Lisa AVE, Marrazzo E, Klinger M, Tinterri C, Testori A. Internal mammary lymph node siliconoma in absence of prosthesis rupture: a case series that raises concern for potential risk of overdiagnosis. Gland Surg 2021;10(7):2123-2129. doi: 10.21037/gs20-860
22. Sacchini G, Borgen PI, Galimberti V, et al. Surgical approach to internal mammary lymph node biopsy. J Am Coll Surg 2001;193:709-13.

23. Bi Z, Zheng WZ, Qiu H, et al. Internal mammary sentinel lymph node biopsy with modified injection technique: A case report. Medicine (Baltimore) 2017;96:e9466.

24. Long H, Lin Z, Situ D, et al. Stage migration and therapy modification after thoracoscopic internal mammary lymph node dissection in breast cancer patients. Breast 2011;20:129-33.

25. Mineo TC, Tamburrini A, Perroni G, et al. 1000 cases of tubeless video-assisted thoracic surgery at the Rome Tor Vergata University. Future Oncol 2016;12:13-8. 